\title{
Optimizing hydroxychloroquine dosing regimen for treatment of pediatric patients with coronavirus disease 2019 using Monte Carlo simulation
}

\author{
Lalitphat Treerattanapun ${ }^{1,2}$, Suwida Tangtrakultham ${ }^{1}$, Nattapong Tidwong 3 , Preecha Montakantikul ${ }^{*}$ \\ 1 Department of Pharmacy, Faculty of Pharmacy, Mahidol University, Bangkok, Thailand \\ 2 Pharmacy Department, Faculty of Medicine Siriraj Hospital, Mahidol University, Bangkok, Thailand \\ 3 Department of Pharmaceutical Care, Faculty of Pharmacy, Chiang Mai University, Chiang Mai, Thailand
}

\begin{abstract}
Hydroxychloroquine may be used to treat COVID-19 infections when remdesivir is unavailable. There is currently no hydroxychloroquine dosage regimen for pediatrics with COVID-19 infections. We aimed to determine the optimal dosage regimen needed to rapidly achieve pharmacokinetic and pharmacodynamic (PKPD) targets for virological clearance in pediatrics. A 10,000-subject Monte Carlo simulation was performed to calculate probabilities of efficacy and safety attainment, using allometrically scaled PKPD targets based on published adult pharmacokinetic studies. Allometric scaling of hydroxychloroquine clearance was also performed. The simulation predicted the probability of target attainment (PTA) of each dosage regimen to achieve an $80 \%$ PTA and $80 \%$ cumulative fraction of response, with $<10 \%$ PTA for toxicity. The loading dosage of $6 \mathrm{mg} / \mathrm{kg} / \mathrm{dose}$, four times daily for 2 days, was found to provide rapid virological clearance with a high PTA $(92.2 \%)$ within 2 days of treatment. Maintenance dosage of $3.25 \mathrm{mg} / \mathrm{kg} / \mathrm{dose}$, three times daily for the next 8 days, achieved the appropriate plasma hydroxychloroquine level until treatment cessation, with a PTA $>80 \%$. As to safety, this dosage regimen achieved a PTA $<10 \%$ of the safety target, giving a probability of cardiotoxicity of $<0.01 \%$. The optimal hydroxychloroquine regimen is the loading dosage of $6 \mathrm{mg} / \mathrm{kg} / \mathrm{dose}$, four times daily for 2 days, followed by maintenance dosage of $3.25 \mathrm{mg} / \mathrm{kg} /$ dose, three times daily, on days 3-10. This regimen achieves virological clearance of COVID-19 and low cardiotoxicity in pediatrics. However, clinical studies are needed to confirm its efficacy and safety.
\end{abstract}

\section{Keywords:}

COVID-19, Hydroxychloroquine, Monte Carlo simulation, Pediatrics

\section{INTRODUCTION}

Coronavirus disease 2019 (COVID-19) is an infectious disease that causes acute respiratory illness. It was first reported in Wuhan, China. The virus has spread rapidly around the world. Epidemiological studies performed in children found that most children infected with COVID-19 were asymptomatic or had mild symptoms. However, about $10 \%$ developed severe symptoms, needed oxygen support, and were admitted to critical care units ${ }^{1}$. The most common route of coronavirus transmission to children is via household contact ${ }^{2}$. No specific drugs have been approved to treat COVID-19 infections in children. Many clinical trials of drugs for the treatment of COVID-19 have been conducted. Some drugs were proven their efficacy for adult patients, but none as yet demonstrated satisfactory outcomes for children $^{3-9}$. Analyses of in vitro data for hydroxychloroquine, an analogue of chloroquine, showed that it effectively inhibited coronavirus replication ${ }^{10-12}$. Hydroxychloroquine blocks coronaviral entry by obstructing glycosylation in the host receptor, thereby viral replication is inhibited by increasing endosomal $\mathrm{pH}$. Hydroxychloroquine also has an immunomodulatory effect by decreasing

\section{*Corresponding author:}

*Preecha Montakantikul preecha.mon@mahidol.ac.th 
cytokine production and inhibiting the activity of lysosomes in host cells; together, these actions may reduce the severity of COVID-19 $13-15$.

Currently, there is no recommended optimal dose nor duration of hydroxychloroquine for the treatment of COVID-19 in pediatric patients. In contrast, several dosage regimens for adults had been proposed. For instance, an in vitro study by Yao et al. ${ }^{10}$ suggested that the loading dose of $400 \mathrm{mg}$ of hydroxychloroquine sulfate should be given orally twice on day 1 , followed by maintenance dose of $200 \mathrm{mg}$ twice daily on days 2 to 5. In comparison, the clinical trial by Gautret and colleagues $^{3}$ used oral hydroxychloroquine sulfate dosage of $200 \mathrm{mg}$, three times a day for ten days.

The Multicenter Initial Guidance on Use of Antivirals for Children with Coronavirus Disease $2019^{16}$ suggests that antivirals be used for children who have severe or critical COVID-19. However, the guidance document suggested that hydroxychloroquine could be used in children who were not candidates for remdesivir, or when remdesivir was unavailable. The suggested loading dose of hydroxychloroquine sulfate for pediatrics (endorsed by the Pediatric Infectious Diseases Society) is $13 \mathrm{mg} / \mathrm{kg}$ (maximum, $800 \mathrm{mg}$ ), followed by $6.5 \mathrm{mg} / \mathrm{kg}$ (maximum, $400 \mathrm{mg}$ ) at 6,24, and 48 hours (the duration can be extended for up to 5 days on a case-by-case basis) ${ }^{16}$. This dosage was based on the simulation that achieved the greatest area under the concentration-time curve from 0 to $24 \mathrm{~h}$ (AUC0-24) for acute uncomplicated malaria.

To date, no pediatric dosage regimen has been evaluated via clinical studies of COVID-19. Moreover, the published data from adult clinical studies had many limitations, and there were conflicting results for the various dosage regimens investigated. The objective of the present study was to determine the optimal dosage regimen of hydroxychloroquine needed for rapid and safe virological clearance in pediatrics infected with COVID-19. A Monte Carlo simulation was employed for this purpose.

\section{MATERIALS AND METHODS}

Table 1. Allometric scaling of population pharmacokinetic parameters.

\begin{tabular}{|c|c|c|c|c|c|c|}
\hline Body weight (kg) & $\mathrm{Ka}\left(\mathrm{hr}^{-1}\right)$ & ALAG (hr) & Vc (L) & Vp (L) & $\mathbf{Q}(\mathrm{L} / \mathrm{hr})$ & CL/F (L/hr) \\
\hline 15 & 1.150 & 0.389 & 93.643 & 297.857 & 14.204 & 3.433 \\
\hline 20 & 1.150 & 0.389 & 124.857 & 397.143 & 17.625 & 4.260 \\
\hline 30 & 1.150 & 0.389 & 187.286 & 595.714 & 23.889 & 5.774 \\
\hline 35 & 1.150 & 0.389 & 218.500 & 695.0 & 26.817 & 6.481 \\
\hline \multicolumn{7}{|c|}{$\begin{array}{l}\text { Random effect } \\
\text { Inter-individual variability }(\% \mathrm{CV})\end{array}$} \\
\hline ALAG & 18.947 & & & & & \\
\hline $\mathrm{Vc}$ & 48.166 & & & & & \\
\hline $\mathrm{Vp}$ & 84.558 & & & & & \\
\hline $\mathrm{CL} / \mathrm{F}$ & 40.125 & & & & & \\
\hline
\end{tabular}

$\mathrm{ALAG}=$ absorption lag time; $\mathrm{CL} / \mathrm{F}=$ apparent clearance; $\mathrm{ka}=$ absorption rate constant; $\mathrm{Q}=$ intercompartmental clearance; $\mathrm{Vc}=\mathrm{central}$ volume of distribution; $\mathrm{Vp}=$ peripheral volume of distribution

\subsection{Pharmacokinetics parameters}

All published population pharmacokinetics of hydroxychloroquine were reviewed. COVID-19 is an acute illness, and short-term usage of antiviral is needed. We therefore identified the pharmacokinetic (PK) parameters in a non-steady state, using a two-compartment linear model with first-order absorption and lag time. The PK-parameter data from 22 healthy subjects and 69 malaria patients were used for our Monte Carlo simula$\operatorname{tion}^{17}$. The mean values of the population-PK parameters and the interindividual variations were used to create individual PK profiles (Table 1). To perform our pediatric simulation, the values of the clearance and intercompartment clearance of hydroxychloroquine from the adult studies were allometrically scaled for weight by an exponent of 0.75 , which is typically used to scale size-related changes in plasma clearance from adults to children $^{18-20}$. However, the volume of distribution $(\mathrm{Vd})$ in the central and peripheral compartments of our model were scaled with an exponent of 1 (Table 1). Allometry based theory indicates that the volume of distribution will be proportional to body weight ${ }^{21}$. Even though Marahaj et al. ${ }^{22}$ found a relationship between $\mathrm{Vd}$ and serum protein, pediatric body composition, including body water and serum protein, typically reaches adult levels by 10-12 months of age ${ }^{23}$. Consequently, the scaling value that we used in our study might be accurate in our study age range. The pediatric weights we used to represent children aged 2-12 years were 15, 20,30, and $35 \mathrm{~kg}$.

\subsection{Pharmacodynamic parameters}

We reviewed the pharmacodynamic parameters of in vitro and clinical studies on COVID-19 patients. To establish the PKPD targets, data were used from an open-label, non-randomized, clinical trial ${ }^{3}$ that detailed virological clearance and serum hydroxychloroquine levels. The average serum concentration of hydroxychloroquine in pneumonia or bronchitis patients who had negative polymerase chain reaction (PCR) of distribution; $\mathrm{V}=$ peripheral volume of distribution 
Table 2. Hydroxychloroquine dosage regimens for simulations.

\section{Dosage regimen}

D1: $6.5 \mathrm{mg} / \mathrm{kg} /$ dose BID

Reference

D2-D10: $3.25 \mathrm{mg} / \mathrm{kg} /$ dose BID

D1-D10: $3.25 \mathrm{mg} / \mathrm{kg} /$ dose TID

Yao et al. ${ }^{10}$

$13 \mathrm{mg} / \mathrm{kg} / \mathrm{dose}$; then, $6.5 \mathrm{mg} / \mathrm{kg} / \mathrm{dose}$ at $6,24,48,72,96 \mathrm{hrs}$

D1: $10 \mathrm{mg} / \mathrm{kg} / \mathrm{dose}$ BID

Gautret et al. ${ }^{3}$

D2-D10: $6.5 \mathrm{mg} / \mathrm{kg} / \mathrm{dose}$ BID

BW 5-24 kg: D1: $6 \mathrm{mg} / \mathrm{kg} /$ dose BID

Chiotos et al. (endorsed by the PIDS) ${ }^{16}$

D2-D10: $2.5 \mathrm{mg} / \mathrm{kg} /$ dose BID

BW 25-49 kg: D1: $6 \mathrm{mg} / \mathrm{kg} /$ dose BID

D2-D10: $3 \mathrm{mg} / \mathrm{kg} / \mathrm{dose}$ BID

D1-2: $6.5 \mathrm{mg} / \mathrm{kg} /$ dose TID Our study regimens

D3-D10: $3.25 \mathrm{mg} / \mathrm{kg} /$ dose TID

D1-2: $6.5 \mathrm{mg} / \mathrm{kg} /$ dose QID

D3-D10: $3.25 \mathrm{mg} / \mathrm{kg} /$ dose TID

D1-2: $6 \mathrm{mg} / \mathrm{kg} / \mathrm{dose}$ QID

D3-D10: $3 \mathrm{mg} / \mathrm{kg} / \mathrm{dose}$ TID

D1-2: $6 \mathrm{mg} / \mathrm{kg} / \mathrm{dose}$ QID

D3-D10: $3.25 \mathrm{mg} / \mathrm{kg} /$ dose TID

D1-2: $6.5 \mathrm{mg} / \mathrm{kg} / \mathrm{dose}$ QID

D3-D10: $3.25 \mathrm{mg} / \mathrm{kg} /$ dose BID

D1-2: $10 \mathrm{mg} / \mathrm{kg} /$ dose BID

D3-D10 $3.25 \mathrm{mg} / \mathrm{kg} / \mathrm{dose}$ BID

$\mathrm{BID}=$ twice daily; PIDS= Pediatric Infectious Diseases Society; $\mathrm{QID}=$ four times daily; TID= three times daily

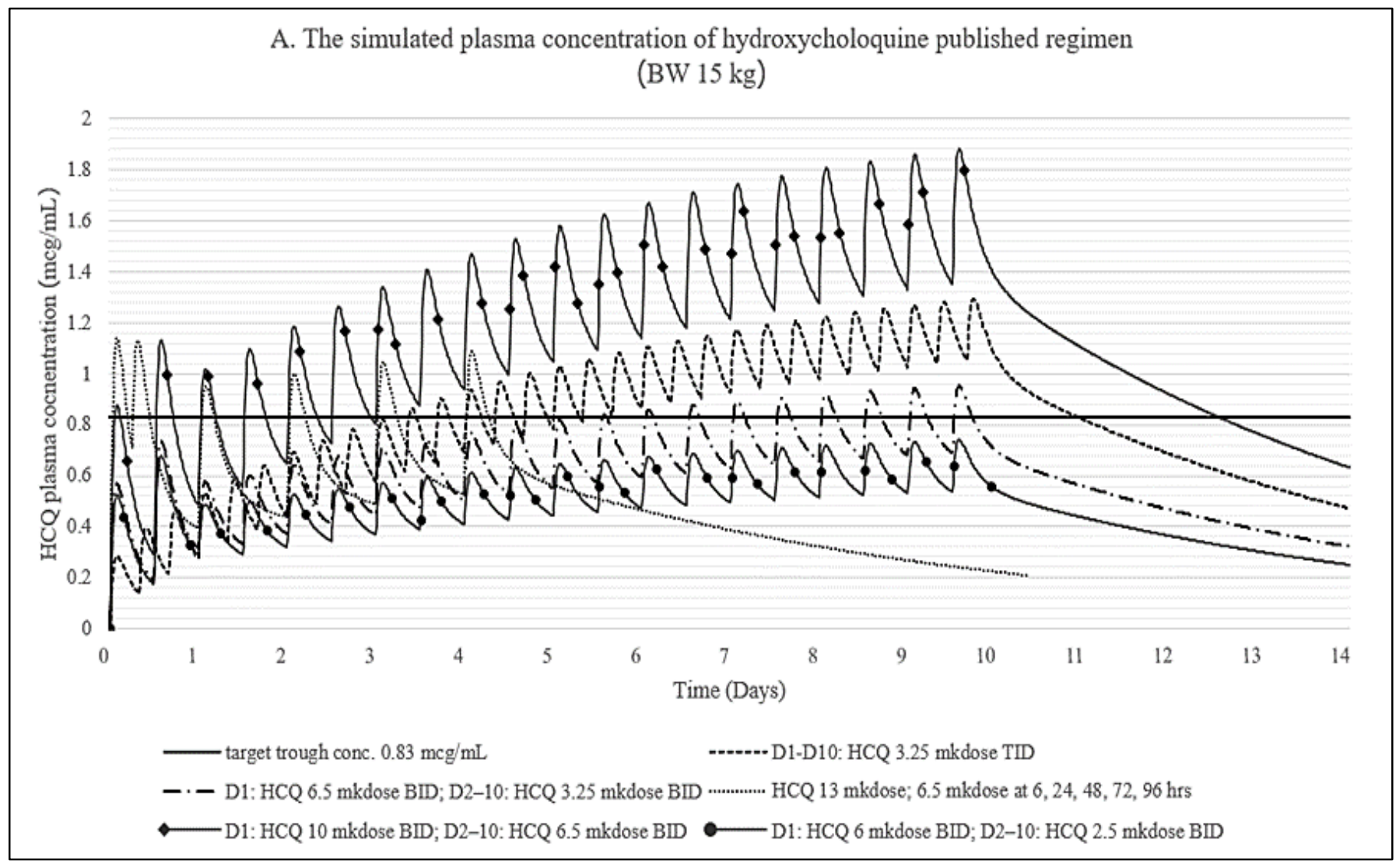

Figures 1A. Simulated HCQ plasma concentration of each published dosage regimen for various body weights. A]. For body weight $15 \mathrm{~kg}$. B]. For body weight $20 \mathrm{~kg}$. C]. For body weight $30 \mathrm{~kg}$. D]. For body weight $35 \mathrm{~kg}$. 


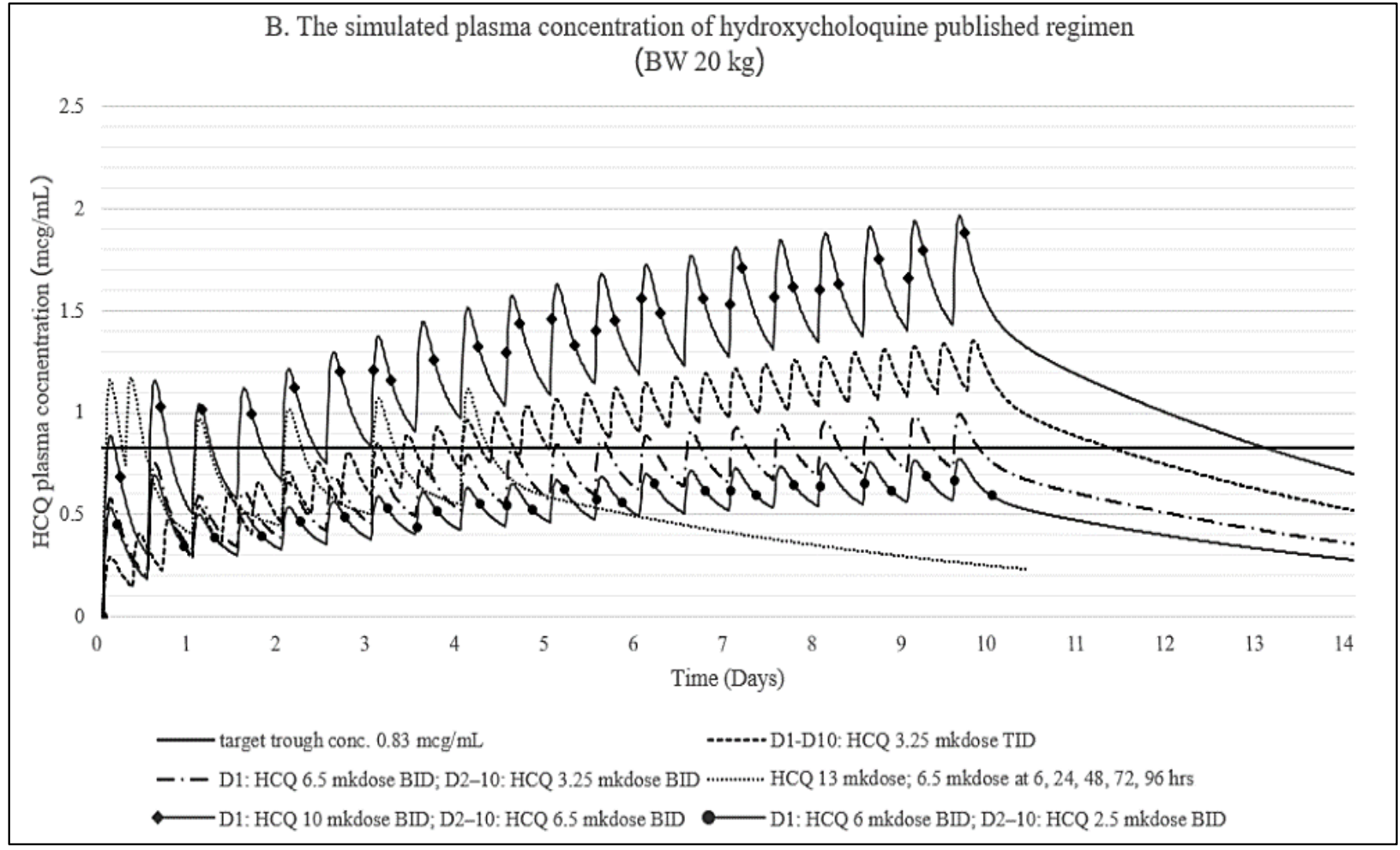

Figures 1B. Simulated HCQ plasma concentration of each published dosage regimen for various body weights. A]. For body weight $15 \mathrm{~kg}$. B]. For body weight $20 \mathrm{~kg}$. C]. For body weight $30 \mathrm{~kg}$. D]. For body weight $35 \mathrm{~kg}$.

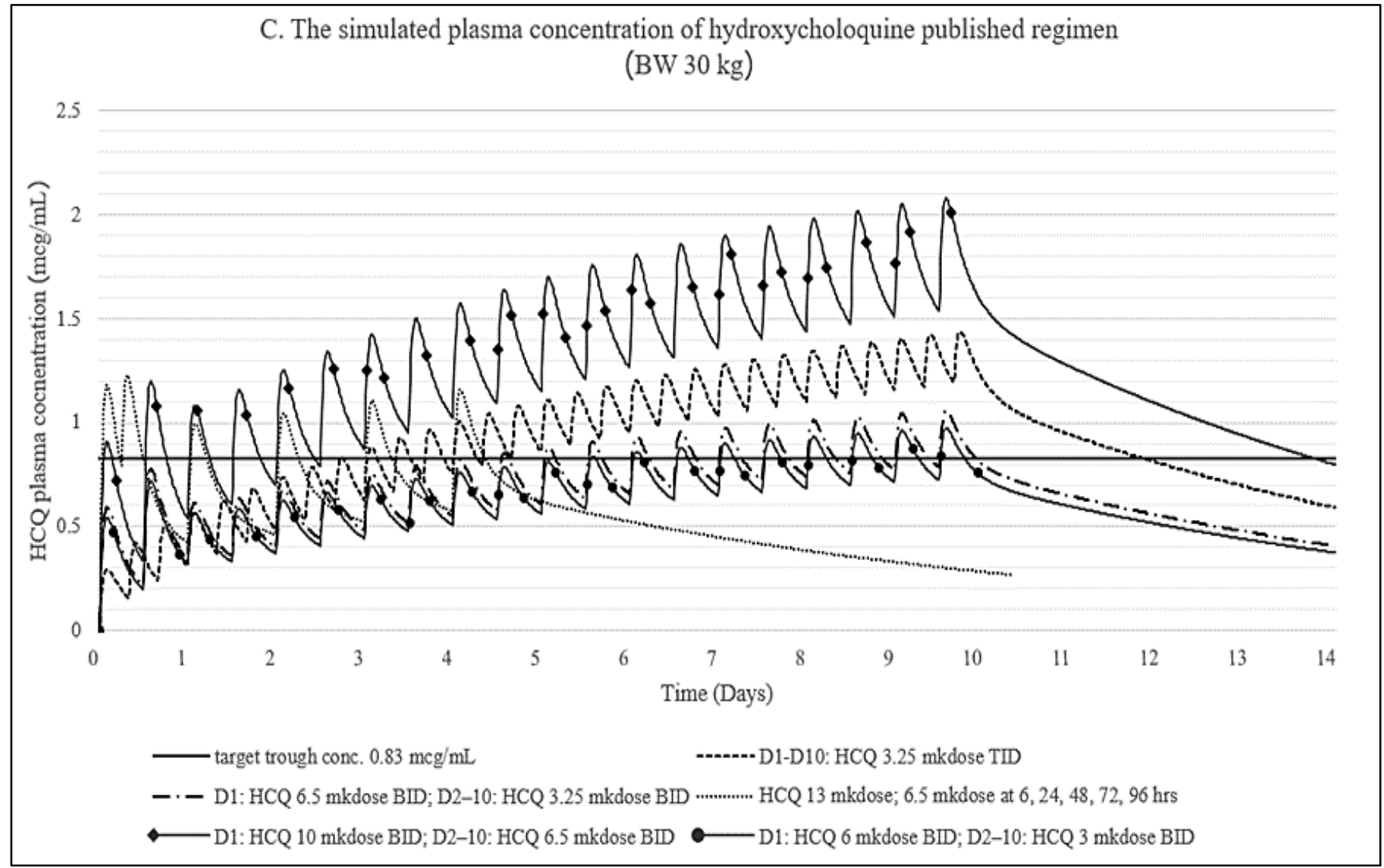

Figures 1C. Simulated HCQ plasma concentration of each published dosage regimen for various body weights. A]. For body weight $15 \mathrm{~kg}$. B]. For body weight $20 \mathrm{~kg}$. C]. For body weight $30 \mathrm{~kg}$. D]. For body weight $35 \mathrm{~kg}$. 


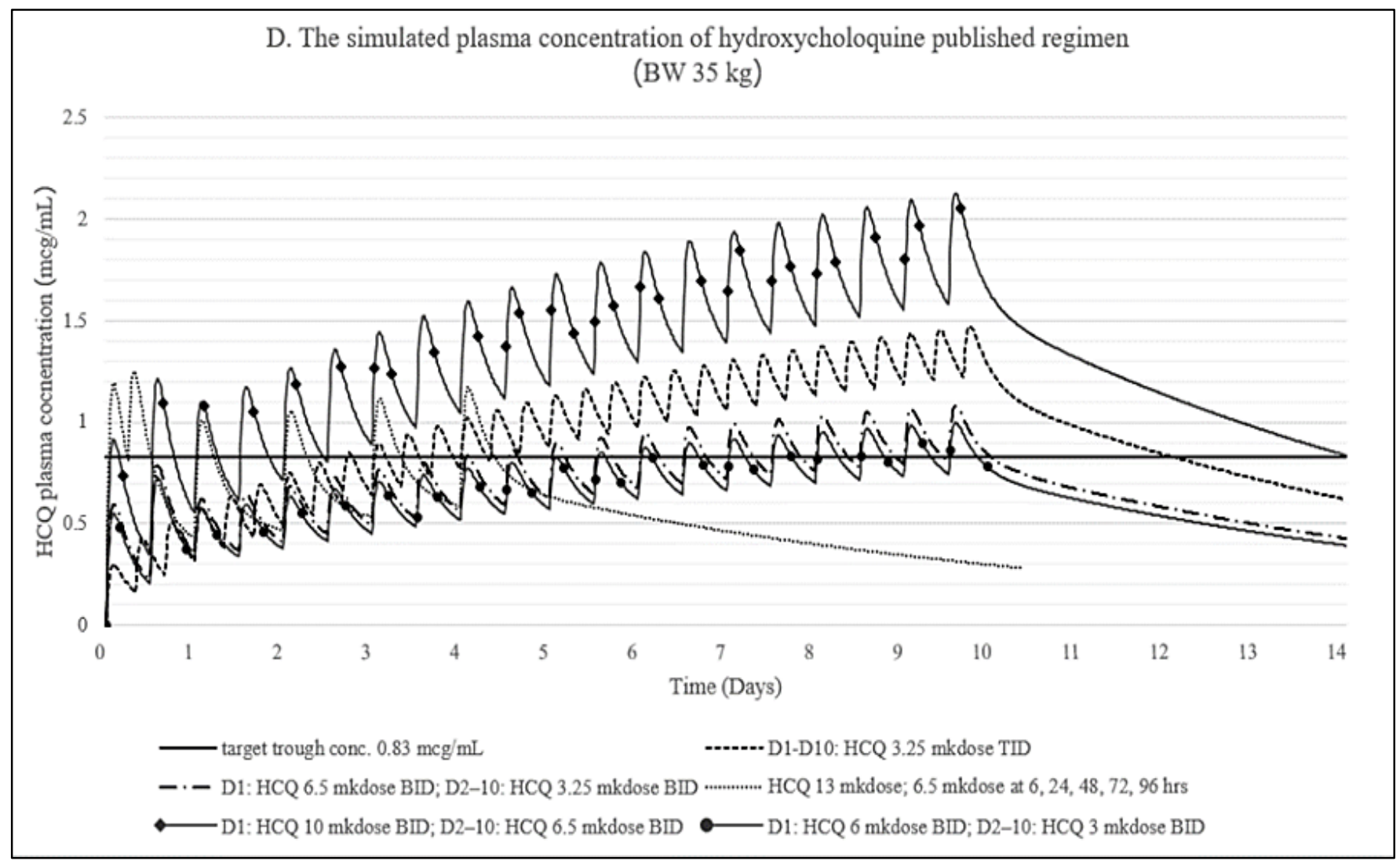

Figures 1D. Simulated HCQ plasma concentration of each published dosage regimen for various body weights. A]. For body weight $15 \mathrm{~kg}$. B]. For body weight $20 \mathrm{~kg}$. C]. For body weight $30 \mathrm{~kg}$. D]. For body weight $35 \mathrm{~kg}$.

results in nasopharyngeal samples at day 6 was 0.83 $\mathrm{mcg} / \mathrm{mL}$. Therefore, we decided to use $0.83 \mathrm{mcg} / \mathrm{mL}$ as the plasma trough level of hydroxychloroquine at any day of treatment as the minimum PKPD target for virological clearance. As to the maximum PKPD target for our study, pediatric overdoses report of hydroxychloroquine is limited and there is no established toxic or lethal plasma level of hydroxychloroquine in adults and pediatrics. Toxic plasma hydroxychloroquine levels were reported to range from $0.635 \mathrm{mcg} / \mathrm{mL}$ (mild gastrointestinal disturbance) ${ }^{24}$ to $9.87 \mathrm{mcg} / \mathrm{mL}$ (hypotension, and ventricular tachyarrhythmia in 18 -years old female $)^{25}$. Thus, we drew upon data from a clinical PK/PD-QTc model simulation by Garcia-Cremades et al., ${ }^{26}$ which they used to investigate the risk of QTc prolongation associated with hydroxychloroquine. Their model was created using a previously published PK-QTc model for high-dose chloroquine in children ${ }^{27}$. They found that mean plasma hydroxychloroquine concentrations higher than $2.6 \mathrm{mcg} / \mathrm{mL}$ were associated with $>1 \%$ of patients experiencing increases of $>60 \mathrm{msec}$ QTc while on treatment. Consequently, we used a peak hydroxychloroquine level of $2.6 \mathrm{mcg} / \mathrm{mL}$ as the cut point to identify toxicity.

Probabilities of efficacy and safety attainment were calculated via a 10,000-pediatric-virtual-subject Monte Carlo simulation (Crystal Ball version 2017; Decisioneering Inc., Denver, Colo., USA). The allome- trically scaled PKPD targets employed were based on published adult PK studies. The simulation utilized 11 hydroxychloroquine regimens. They were drawn from in vitro data ${ }^{10}$, a clinical trial ${ }^{3}$, antiviral-use guidance for children with COVID-19 $19^{16}$, Thai COVID-19 guidelines, pediatric dosing simulation data ${ }^{27}$, and regimens that we specially developed (Table 2).

\section{RESULTS}

The results of \%PTA of the simulation regimens are listed in Tables 3.1-3.2 and simulated hydroxychloroquine plasma concentration profiles are shown in Figure 1 and Figure 2. Six regimens achieved the target plasma trough level (Ctrough $>0.83 \mathrm{mcg} / \mathrm{mL}$ ) by day 10 of the treatment (regimens 2, 4, 6, 7, 8, and 9). Regimens $4,6,7$, and 9 achieved the therapeutic target for all ages (represented by the selected pediatric weights). Regimens 7 and 9 reached the therapeutic target within 2 days.

The published dosage regimens which achieved a probability of target attainment (PTA) $>80 \%$ by Day 10 of treatment were regimens 2 and 4 . Regimen 2 was used in the clinical study by Gautret et al., ${ }^{3}$ while regimen 4 was obtained from the Thai COVID-19 guidelines. Of the regimens we created for the simulation, regimens 6 , 7,8 , and 9 reached the target trough level $(0.83 \mathrm{mcg} / \mathrm{mL})$ and had a \%PTA greater than $80 \%$ by Day 10 of treatment. The PTA values of regimens 6, 7, and 9 were over 
$80 \%$ for all ages. However, only regimens 7 and 9 reached the target plasma trough level within 2 days of treatment. The average percentages of PTA of regimen 7 on Days 2 and 10 of treatment were $95.1 \%$ and $88.9 \%$, respectively. The corresponding values for regimen 9 were $92.2 \%$ and $88.3 \%$.

Due to concerns about possible cardiovascular side effects and toxicity ${ }^{24,28-33}$, the PTA values at plasma concentrations above the target cut point were determined. We assumed that the optimal regimen should have a \%PTA of not more than $10 \%$. The simulation found that regimen 4 had the \%PTA with the highest value at Day 10 of treatment. The average \%PTA of regimen 4 was $26.17 \%$. As to the regimens we developed for the simulation, regimens 6 and 9 each had a \%PTA of not more than $10 \%$ for all treatment courses. Due to

Table 3.1. Probability of target attainment for each hydroxychloroquine regimen and body weight, with targets of $\mathrm{C}_{\text {trough }} \geq 0.83 \mathrm{mcg} / \mathrm{mL}$ for virological clearance of COVID-19.

\begin{tabular}{|c|c|c|c|c|c|c|c|c|}
\hline & $\begin{array}{c}\text { \% PTA } \\
\text { Ctrough }\end{array}$ & Day 1 & Day 2 & Day 3 & Day 5 & Day 7 & Day 9 & Day 10 \\
\hline Regimen1; & $15 \mathrm{~kg}$ & 1.34 & 3.63 & 7.62 & 17.45 & 26.50 & 33.27 & 36.25 \\
\hline D1: HCQ 6.5 mkdose BID; & $20 \mathrm{~kg}$ & 1.38 & 4.30 & 9.54 & 21.03 & 31.14 & 39.13 & 42.48 \\
\hline \multirow[t]{2}{*}{ D2-10: HCQ 3.25 mkdose BID } & $30 \mathrm{~kg}$ & 1.97 & 5.97 & 11.99 & 26.64 & 38.42 & 46.59 & 50.63 \\
\hline & $35 \mathrm{~kg}$ & 1.95 & 6.55 & 13.48 & 29.18 & 41.86 & 50.73 & 54.87 \\
\hline Regimen 2; & $15 \mathrm{~kg}$ & 0.19 & 7.04 & 22.03 & 51.15 & 66.41 & 74.51 & 77.81 \\
\hline \multirow[t]{3}{*}{ HCQ 3.25 mkdose TID } & $20 \mathrm{~kg}$ & 0.21 & 8.25 & 25.65 & 55.93 & 71.04 & 78.63 & 81.32 \\
\hline & $30 \mathrm{~kg}$ & 0.31 & 10.56 & 31.20 & 61.77 & 76.59 & 83.74 & 86.10 \\
\hline & $35 \mathrm{~kg}$ & 0.30 & 10.93 & 32.07 & 63.95 & 78.37 & 85.68 & 87.87 \\
\hline \multirow{4}{*}{$\begin{array}{l}\text { Regimen } 3 \text {; } \\
\text { HCQ } 13 \text { mkdose; } 6.5 \text { mkdose at } 6,24,48 \text {, } \\
72,96 \mathrm{hrs}\end{array}$} & $15 \mathrm{~kg}$ & 8.14 & 9.89 & 12.86 & 19.38 & 3.05 & 0.45 & 0.20 \\
\hline & $20 \mathrm{~kg}$ & 8.90 & 11.44 & 15.35 & 23.04 & 3.79 & 0.60 & 0.26 \\
\hline & $30 \mathrm{~kg}$ & 10.71 & 14.29 & 18.88 & 28.15 & 5.89 & 1.14 & 0.59 \\
\hline & $35 \mathrm{~kg}$ & 11.24 & 15.17 & 20.24 & 30.81 & 6.95 & 1.31 & 0.65 \\
\hline \multirow{4}{*}{$\begin{array}{l}\text { Regimen 4; } \\
\text { D1: HCQ } 10 \text { mkdose BID; D2-10: HCQ } \\
6.5 \text { mkdose BID }\end{array}$} & $15 \mathrm{~kg}$ & 13.84 & 37.47 & 57.83 & 77.69 & 85.71 & 89.47 & 90.76 \\
\hline & $20 \mathrm{~kg}$ & 14.99 & 40.44 & 59.61 & 79.25 & 87.60 & 91.23 & 92.60 \\
\hline & $30 \mathrm{~kg}$ & 18.06 & 45.39 & 65.28 & 84.29 & 90.99 & 94.13 & 95.21 \\
\hline & $35 \mathrm{~kg}$ & 18.65 & 46.10 & 65.73 & 84.49 & 91.54 & 94.68 & 95.67 \\
\hline \multirow{2}{*}{$\begin{array}{l}\text { Regimen 5; } \\
\text { D1: HCQ } 6 \text { mkdose BID; } \\
\text { D2-10: HCQ } 2.5 \text { mkdose BID }\end{array}$} & $15 \mathrm{~kg}$ & 0.58 & 1.20 & 2.61 & 6.18 & 10.30 & 14.07 & 16.03 \\
\hline & $20 \mathrm{~kg}$ & 0.83 & 1.80 & 3.48 & 7.92 & 12.72 & 17.42 & 19.51 \\
\hline \multirow{2}{*}{$\begin{array}{l}\text { Regimen 5; } \\
\text { D1: HCQ } 6 \text { mkdose BID; } \\
\text { D2-10: HCQ } 3 \text { mkdose BID }\end{array}$} & $30 \mathrm{~kg}$ & 1.01 & 3.42 & 7.87 & 19.73 & 29.84 & 37.76 & 41.21 \\
\hline & $35 \mathrm{~kg}$ & 1.19 & 3.91 & 8.76 & 21.28 & 32.63 & 40.88 & 44.77 \\
\hline \multirow{4}{*}{$\begin{array}{l}\text { Regimen 6; } \\
\text { D1-2: HCQ } 6.5 \text { mkdose TID; D3-10: } 3.25 \\
\text { mkdose TID }\end{array}$} & $15 \mathrm{~kg}$ & 17.13 & 66.27 & 64.18 & 71.97 & 76.52 & 79.88 & 81.40 \\
\hline & $20 \mathrm{~kg}$ & 19.73 & 69.82 & 67.42 & 75.61 & 80.36 & 83.32 & 84.88 \\
\hline & $30 \mathrm{~kg}$ & 22.47 & 74.51 & 71.38 & 80.63 & 85.51 & 88.33 & 89.72 \\
\hline & $35 \mathrm{~kg}$ & 24.08 & 77.15 & 73.23 & 82.56 & 87.07 & 89.89 & 91.09 \\
\hline \multirow{4}{*}{$\begin{array}{l}\text { Regimen 7; } \\
\text { D1-2: HCQ } 6.5 \text { mkdose QID; D3-10: } \\
3.25 \text { mkdose TID }\end{array}$} & $15 \mathrm{~kg}$ & 55.58 & 91.79 & 79.59 & 80.80 & 81.64 & 82.72 & 83.46 \\
\hline & $20 \mathrm{~kg}$ & 60.84 & 94.67 & 82.55 & 84.16 & 85.58 & 86.90 & 87.69 \\
\hline & $30 \mathrm{~kg}$ & 70.59 & 96.51 & 84.49 & 87.33 & 89.05 & 90.39 & 91.16 \\
\hline & $35 \mathrm{~kg}$ & 74.16 & 97.39 & 85.76 & 88.90 & 90.95 & 92.51 & 93.23 \\
\hline \multirow{4}{*}{$\begin{array}{l}\text { Regimen 8; } \\
\text { HCQ } 6 \text { mkdose QID; } \\
\text { D3-10: } 3 \text { mkdose TID }\end{array}$} & $15 \mathrm{~kg}$ & 45.28 & 88.54 & 74.75 & 75.10 & 76.00 & 77.17 & 78.17 \\
\hline & $20 \mathrm{~kg}$ & 50.39 & 90.93 & 76.78 & 78.71 & 80.47 & 81.89 & 82.81 \\
\hline & $30 \mathrm{~kg}$ & 59.09 & 94.20 & 80.46 & 83.23 & 85.76 & 87.08 & 87.93 \\
\hline & $35 \mathrm{~kg}$ & 62.77 & 95.24 & 81.80 & 85.18 & 87.48 & 88.75 & 89.62 \\
\hline \multirow{4}{*}{$\begin{array}{l}\text { Regimen 9; } \\
\text { HCQ 6 mkdose QID; } \\
\text { D3-10: } 3.25 \text { mkdose TID }\end{array}$} & $15 \mathrm{~kg}$ & 45.09 & 88.19 & 76.03 & 78.39 & 80.09 & 81.64 & 82.79 \\
\hline & $20 \mathrm{~kg}$ & 51.71 & 91.02 & 79.03 & 82.22 & 84.47 & 86.05 & 86.95 \\
\hline & $30 \mathrm{~kg}$ & 59.56 & 94.44 & 82.48 & 86.6 & 89.01 & 90.45 & 91.37 \\
\hline & $35 \mathrm{~kg}$ & 62.42 & 95.19 & 83.01 & 87.09 & 89.80 & 91.30 & 92.06 \\
\hline \multirow{4}{*}{$\begin{array}{l}\text { Regimen 10; } \\
\text { D1-2: HCQ } 6.5 \text { mkdose QID; D3-10: } \\
\text { 3.25 mkdose BID }\end{array}$} & $15 \mathrm{~kg}$ & 55.90 & 91.98 & 70.15 & 61.44 & 55.63 & 53.31 & 52.90 \\
\hline & $20 \mathrm{~kg}$ & 62.39 & 94.57 & 74.16 & 66.33 & 61.84 & 59.21 & 59.18 \\
\hline & $30 \mathrm{~kg}$ & 70.37 & 96.55 & 76.35 & 72.07 & 69.06 & 68.02 & 67.75 \\
\hline & $35 \mathrm{~kg}$ & 74.02 & 96.99 & 77.32 & 73.34 & 71.14 & 69.87 & 69.76 \\
\hline \multirow{4}{*}{$\begin{array}{l}\text { Regimen 11; } \\
\text { D1-2: HCQ } 10 \text { mkdose BID; D3-10: } 3.25 \\
\text { mkdose BID }\end{array}$} & $15 \mathrm{~kg}$ & 14.33 & 59.83 & 51.13 & 46.95 & 45.68 & 46.09 & 46.78 \\
\hline & $20 \mathrm{~kg}$ & 16.13 & 62.90 & 54.80 & 51.75 & 51.85 & 52.90 & 53.71 \\
\hline & $30 \mathrm{~kg}$ & 18.29 & 66.82 & 58.77 & 58.04 & 58.74 & 60.04 & 61.21 \\
\hline & $35 \mathrm{~kg}$ & 18.18 & 68.66 & 60.04 & 60.85 & 62.18 & 64.17 & 65.29 \\
\hline
\end{tabular}

$\mathrm{BID}=$ twice daily; $\mathrm{C}_{\text {trough }}=$ trough concentration; $\mathrm{HCQ}=$ hydroxychloroquine; $\mathrm{mkdose}=\mathrm{mg} / \mathrm{kg} / \mathrm{dose} ; \mathrm{PTA}=$ probability of target attainment; TID $=$ three times daily 
Table 3.2. Probability of target attainment for each hydroxychloroquine regimen and body weight, with targets of $\mathrm{C}_{\max } \geq 2.6 \mathrm{mcg} / \mathrm{mL}$ for toxicity level.

\begin{tabular}{|c|c|c|c|c|c|c|c|c|}
\hline & $\begin{array}{c}\% \text { PTA } \\
\mathrm{C}_{\max } \\
\end{array}$ & Day 1 & Day 2 & Day 3 & Day 5 & Day 7 & Day 9 & Day 10 \\
\hline Regimen1; & $15 \mathrm{~kg}$ & 0.00 & 0.00 & 0.00 & 0.00 & 0.02 & 0.03 & 0.04 \\
\hline D1: HCQ 6.5 mkdose BID; & $20 \mathrm{~kg}$ & 0.00 & 0.00 & 0.01 & 0.02 & 0.06 & 0.12 & 0.14 \\
\hline \multirow[t]{2}{*}{ D2-10: HCQ 3.25 mkdose BID } & $30 \mathrm{~kg}$ & 0.00 & 0.00 & 0.00 & 0.03 & 0.07 & 0.14 & 0.22 \\
\hline & $35 \mathrm{~kg}$ & 0.00 & 0.00 & 0.01 & 0.06 & 0.11 & 0.22 & 0.25 \\
\hline \multirow{4}{*}{$\begin{array}{l}\text { Regimen } 2 ; \\
\text { HCQ } 3.25 \text { mkdose TID }\end{array}$} & $15 \mathrm{~kg}$ & 0.00 & 0.00 & 0.00 & 0.21 & 0.69 & 1.39 & 1.75 \\
\hline & $20 \mathrm{~kg}$ & 0.00 & 0.00 & 0.00 & 0.27 & 0.89 & 2.09 & 2.83 \\
\hline & $30 \mathrm{~kg}$ & 0.00 & 0.00 & 0.04 & 0.46 & 1.75 & 3.27 & 4.20 \\
\hline & $35 \mathrm{~kg}$ & 0.00 & 0.00 & 0.05 & 0.42 & 1.45 & 3.48 & 4.37 \\
\hline \multirow{4}{*}{$\begin{array}{l}\text { Regimen } 3 ; \\
\text { HCQ } 13 \text { mkdose; } 6.5 \text { mkdose at } 6,24 \text {, } \\
48,72,96 \mathrm{hrs}\end{array}$} & $15 \mathrm{~kg}$ & 0.90 & 0.15 & 0.12 & 0.17 & 0.00 & 0.00 & 0.00 \\
\hline & $20 \mathrm{~kg}$ & 1.31 & 0.18 & 0.15 & 0.28 & 0.00 & 0.00 & 0.00 \\
\hline & $30 \mathrm{~kg}$ & 1.60 & 0.23 & 0.22 & 0.39 & 0.00 & 0.00 & 0.00 \\
\hline & $35 \mathrm{~kg}$ & 1.79 & 0.27 & 0.27 & 0.52 & 0.00 & 0.00 & 0.00 \\
\hline \multirow{4}{*}{$\begin{array}{l}\text { Regimen 4; } \\
\text { D1: HCQ } 10 \text { mkdose BID; D2-10: } \\
\text { HCQ } 6.5 \text { mkdose BID }\end{array}$} & $15 \mathrm{~kg}$ & 0.48 & 0.51 & 1.44 & 5.73 & 11.25 & 16.63 & 18.70 \\
\hline & $20 \mathrm{~kg}$ & 0.59 & 0.65 & 2.36 & 7.77 & 14.35 & 20.43 & 22.96 \\
\hline & $30 \mathrm{~kg}$ & 0.87 & 1.01 & 3.22 & 10.93 & 19.39 & 27.04 & 30.51 \\
\hline & $35 \mathrm{~kg}$ & 0.87 & 1.01 & 3.43 & 11.79 & 21.18 & 29.36 & 32.51 \\
\hline \multirow{2}{*}{$\begin{array}{l}\text { Regimen 5; } \\
\text { D1: HCQ } 6 \text { mkdose BID; } \\
\text { D2-10: HCQ } 2.5 \text { mkdose BID }\end{array}$} & $15 \mathrm{~kg}$ & 0.00 & 0.00 & 0.00 & 0.00 & 0.00 & 0.00 & 0.00 \\
\hline & $20 \mathrm{~kg}$ & 0.00 & 0.00 & 0.00 & 0.00 & 0.00 & 0.00 & 0.01 \\
\hline \multirow{2}{*}{$\begin{array}{l}\text { Regimen 5; } \\
\text { D1: HCQ } 6 \text { mkdose BID; } \\
\text { D2-10: HCQ } 3 \text { mkdose BID } \\
\end{array}$} & $30 \mathrm{~kg}$ & 0.00 & 0.00 & 0.00 & 0.00 & 0.00 & 0.05 & 0.06 \\
\hline & $35 \mathrm{~kg}$ & 0.00 & 0.00 & 0.00 & 0.01 & 0.05 & 0.08 & 0.12 \\
\hline \multirow{4}{*}{$\begin{array}{l}\text { Regimen 6; } \\
\text { D1-2: HCQ } 6.5 \text { mkdose TID; D3-10: } \\
\text { 3.25 mkdose TID }\end{array}$} & $15 \mathrm{~kg}$ & 0.03 & 1.93 & 0.86 & 1.27 & 1.91 & 2.70 & 3.20 \\
\hline & $20 \mathrm{~kg}$ & 0.08 & 2.63 & 1.28 & 1.89 & 2.92 & 3.98 & 4.54 \\
\hline & $30 \mathrm{~kg}$ & 0.09 & 3.31 & 1.74 & 2.79 & 4.28 & 6.04 & 6.75 \\
\hline & $35 \mathrm{~kg}$ & 0.13 & 3.59 & 2.01 & 3.12 & 4.82 & 6.97 & 7.97 \\
\hline \multirow{4}{*}{$\begin{array}{l}\text { Regimen 7; } \\
\text { D1-2: HCQ } 6.5 \text { mkdose QID; D3-10: } \\
\text { 3.25 mkdose TID }\end{array}$} & $15 \mathrm{~kg}$ & 0.43 & 10.13 & 6.46 & 3.42 & 3.53 & 4.13 & 4.39 \\
\hline & $20 \mathrm{~kg}$ & 0.46 & 11.36 & 7.23 & 4.27 & 4.75 & 5.49 & 5.71 \\
\hline & $30 \mathrm{~kg}$ & 0.62 & 13.41 & 8.89 & 5.94 & 6.67 & 7.81 & 8.44 \\
\hline & $35 \mathrm{~kg}$ & 0.90 & 15.64 & 10.25 & 7.31 & 8.34 & 9.77 & 10.49 \\
\hline \multirow{4}{*}{$\begin{array}{l}\text { Regimen 8; } \\
\text { HCQ } 6 \text { mkdose QID; } \\
\text { D3-10: } 3 \text { mkdose TID }\end{array}$} & $15 \mathrm{~kg}$ & 0.09 & 6.18 & 3.68 & 1.74 & 1.91 & 2.10 & 2.34 \\
\hline & $20 \mathrm{~kg}$ & 0.15 & 7.41 & 4.65 & 2.45 & 2.58 & 3.08 & 3.30 \\
\hline & $30 \mathrm{~kg}$ & 0.26 & 8.91 & 5.61 & 3.67 & 4.22 & 4.96 & 5.50 \\
\hline & $35 \mathrm{~kg}$ & 0.45 & 10.42 & 6.74 & 4.41 & 5.16 & 6.27 & 6.80 \\
\hline \multirow{4}{*}{$\begin{array}{l}\text { Regimen 9; } \\
\text { HCQ 6 mkdose QID; } \\
\text { D3-10: } 3.25 \text { mkdose TID }\end{array}$} & $15 \mathrm{~kg}$ & 0.15 & 6.02 & 4.02 & 2.20 & 2.43 & 3.05 & 3.43 \\
\hline & $20 \mathrm{~kg}$ & 0.29 & 8.39 & 6.02 & 3.71 & 4.17 & 5.16 & 5.51 \\
\hline & $30 \mathrm{~kg}$ & 0.53 & 9.51 & 6.63 & 5.49 & 6.65 & 8.05 & 8.83 \\
\hline & $35 \mathrm{~kg}$ & 0.37 & 9.89 & 6.94 & 5.56 & 7.08 & 8.27 & 9.01 \\
\hline \multirow{4}{*}{$\begin{array}{l}\text { Regimen 10; } \\
\text { D1-2: HCQ } 6.5 \text { mkdose QID; D3-10: } \\
\text { 3.25 mkdose BID }\end{array}$} & $15 \mathrm{~kg}$ & 0.34 & 9.85 & 6.28 & 1.14 & 0.66 & 0.52 & 0.49 \\
\hline & $20 \mathrm{~kg}$ & 0.63 & 12.12 & 7.71 & 1.77 & 1.03 & 0.87 & 0.83 \\
\hline & $30 \mathrm{~kg}$ & 0.76 & 14.17 & 9.26 & 2.69 & 1.57 & 1.30 & 1.28 \\
\hline & $35 \mathrm{~kg}$ & 0.78 & 14.60 & 9.51 & 2.98 & 1.90 & 1.55 & 1.55 \\
\hline \multirow{4}{*}{$\begin{array}{l}\text { Regimen 11; } \\
\text { D1-2: HCQ } 10 \text { mkdose BID; D3-10: } \\
3.25 \text { mkdose BID }\end{array}$} & $15 \mathrm{~kg}$ & 0.49 & 6.17 & 0.75 & 0.14 & 0.19 & 0.22 & 0.24 \\
\hline & $20 \mathrm{~kg}$ & 0.61 & 7.11 & 1.04 & 0.40 & 0.43 & 0.47 & 0.50 \\
\hline & $30 \mathrm{~kg}$ & 0.96 & 9.05 & 1.65 & 0.76 & 0.75 & 0.82 & 0.84 \\
\hline & $35 \mathrm{~kg}$ & 0.86 & 8.75 & 1.54 & 0.68 & 0.72 & 0.80 & 0.86 \\
\hline
\end{tabular}

$\mathrm{BID}=$ twice daily $; \mathrm{C}_{\max }=$ maximum concentration; $\mathrm{HCQ}=$ hydroxychloroquine; $\mathrm{mkdose}=\mathrm{mg} / \mathrm{kg} / \mathrm{dose} ; \mathrm{PTA}=$ probability of target attainment; $\mathrm{TID}=$ three times daily 


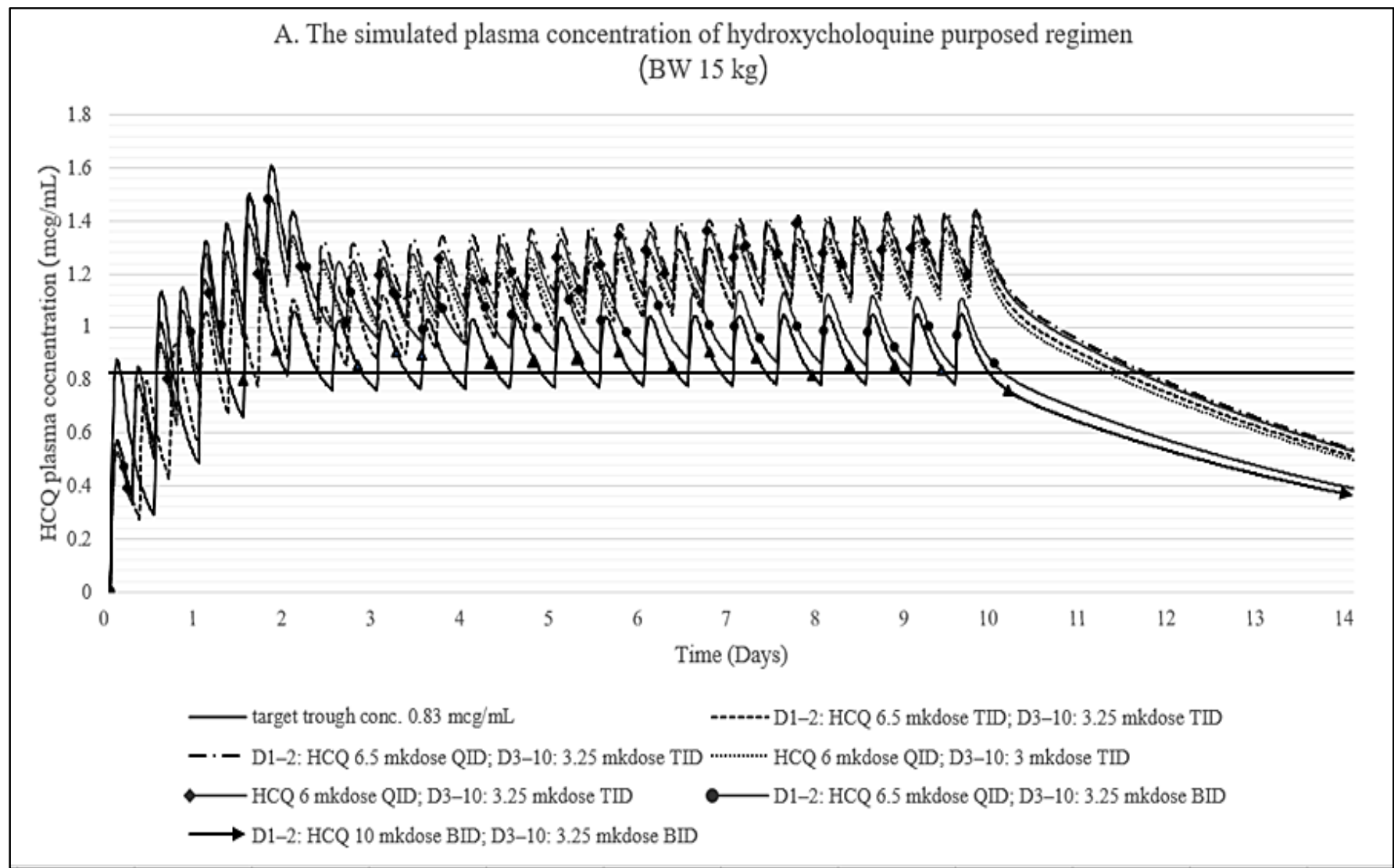

Figures 2A. Simulated HCQ plasma concentration for each purposed dosage regimen for various body weights. A]. For body weight $15 \mathrm{~kg}$. B]. For body weight $20 \mathrm{~kg}$. C]. For body weight $30 \mathrm{~kg}$. D]. For body weight $35 \mathrm{~kg}$.

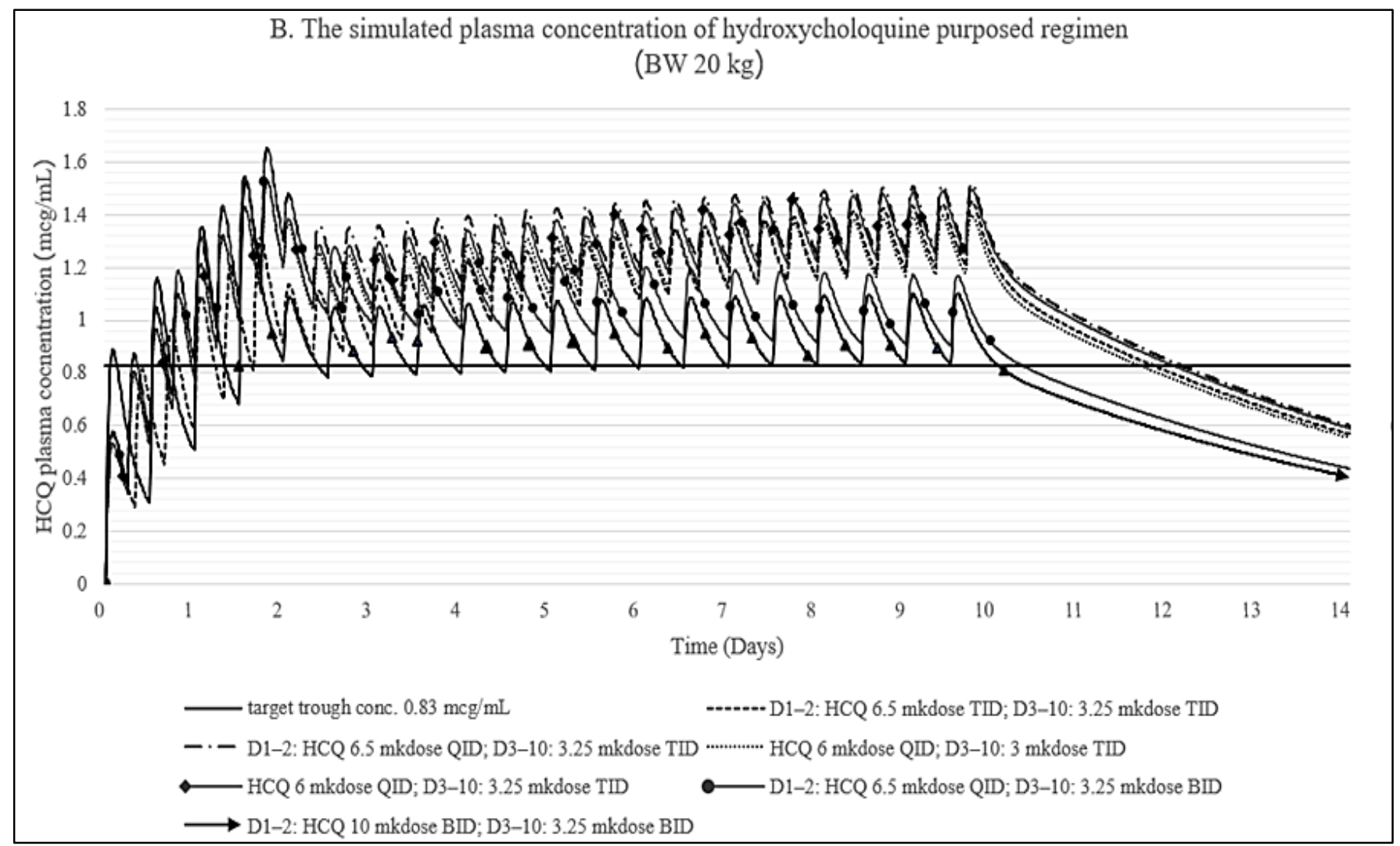

Figures 2B. Simulated HCQ plasma concentration for each purposed dosage regimen for various body weights. A]. For body weight $15 \mathrm{~kg}$. B]. For body weight $20 \mathrm{~kg}$. C]. For body weight $30 \mathrm{~kg}$. D]. For body weight $35 \mathrm{~kg}$. 


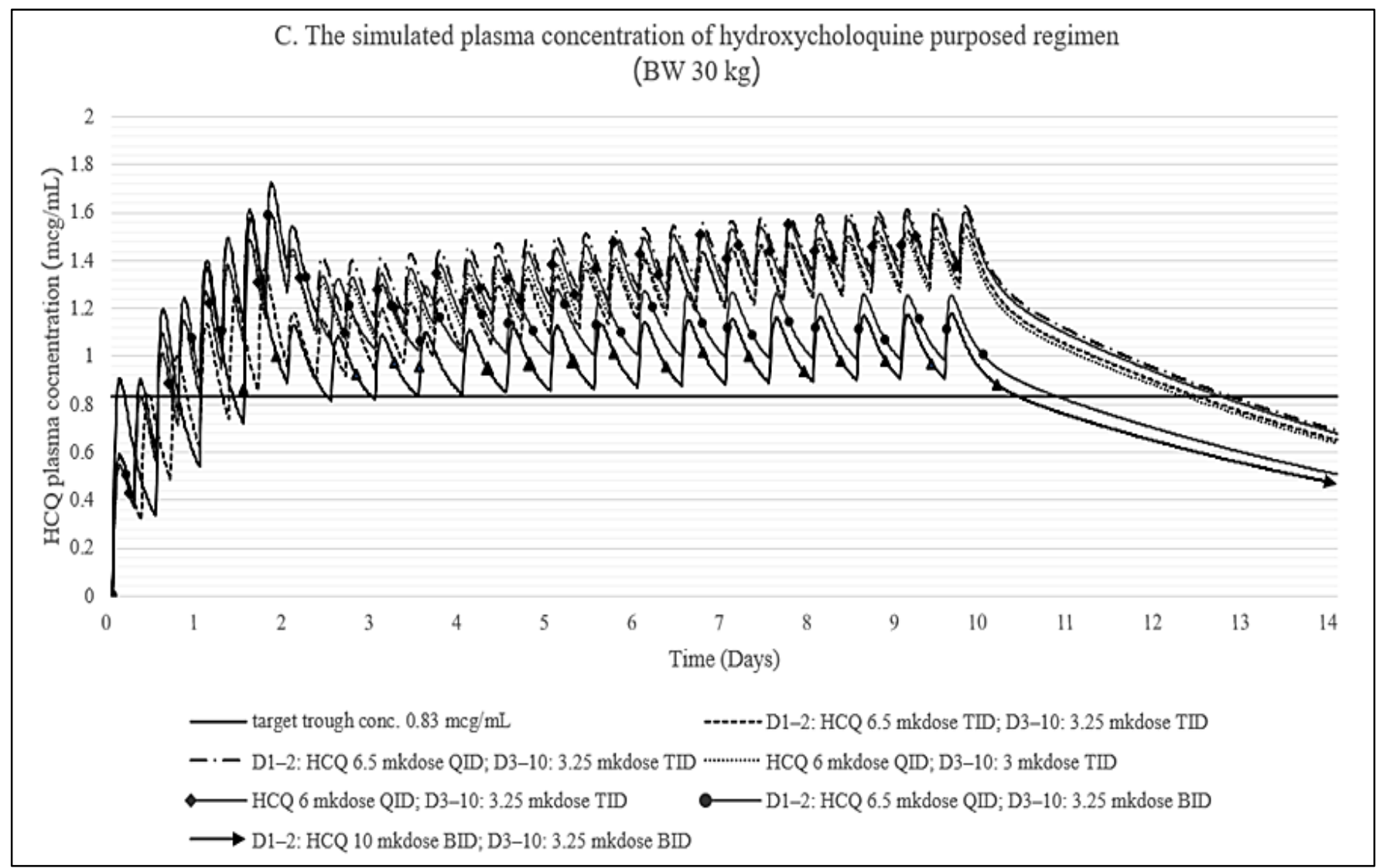

Figures 2C. Simulated HCQ plasma concentration for each purposed dosage regimen for various body weights. A]. For body weight $15 \mathrm{~kg}$. B]. For body weight $20 \mathrm{~kg}$. C]. For body weight $30 \mathrm{~kg}$. D]. For body weight $35 \mathrm{~kg}$.

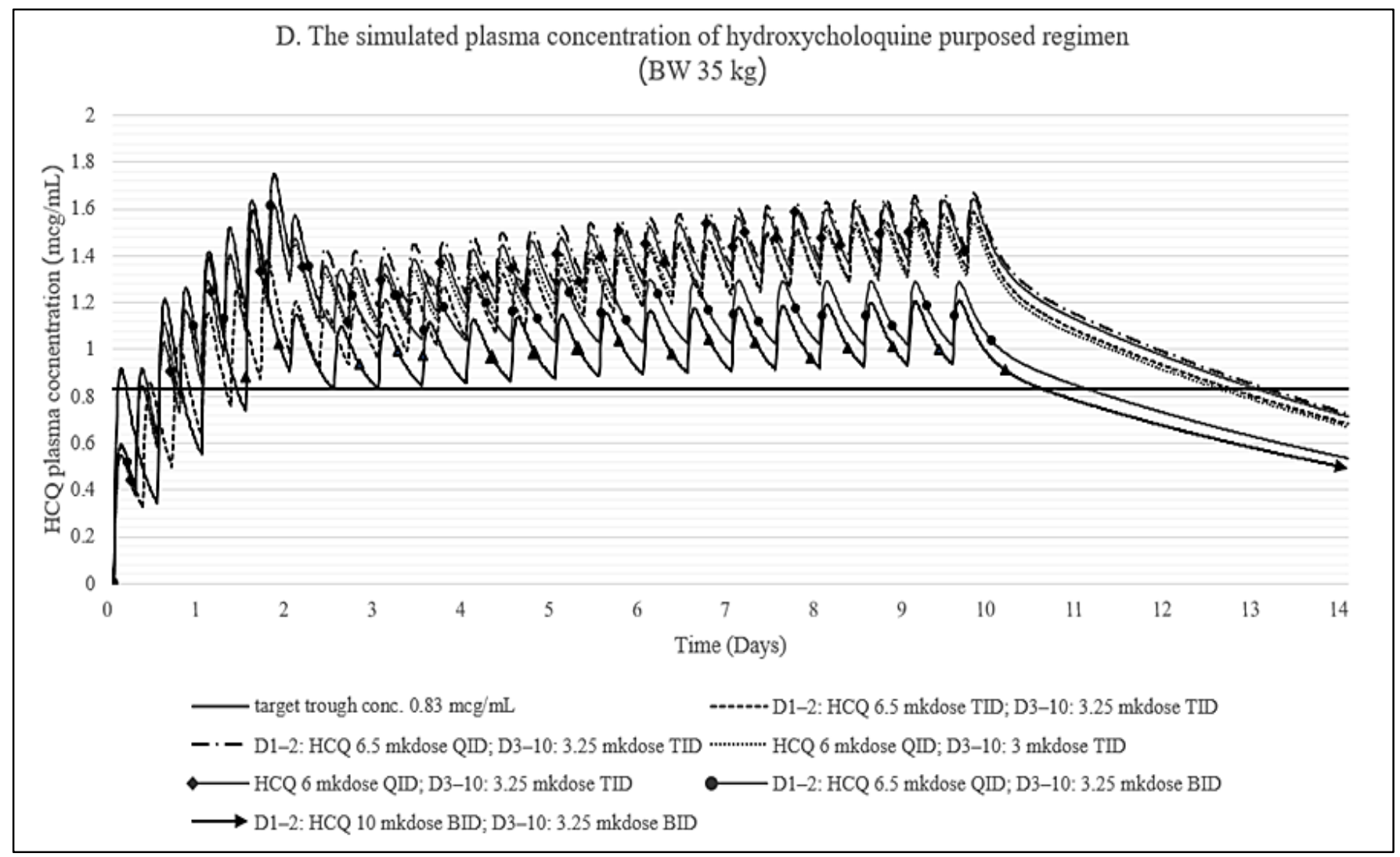

Figures 2D. Simulated HCQ plasma concentration for each purposed dosage regimen for various body weights. A]. For body weight $15 \mathrm{~kg}$. B]. For body weight $20 \mathrm{~kg}$. C]. For body weight $30 \mathrm{~kg}$. D]. For body weight $35 \mathrm{~kg}$. 
their high loading dosages on the first 2 days $(6.5 \mathrm{mg} /$ $\mathrm{kg} /$ dose every 6 hours), the \%PTA values of regimens 7 and 10 exceeded $10 \%$ on Day $2(12.6 \%$ and $12.7 \%$, respectively). However, the \% PTA values began to decline on day 3 .

\section{DISCUSSION}

In the absence of an approved dosage regimen of hydroxychloroquine for the treatment of pediatric COVID-19 patients, our simulation set out to determine a specific regimen. As part of our work, the results of recent efforts to develop a hydroxychloroquine dosage regimen for adults were examined. Regimen 1 in our study was the dosage that had been used in an in vitro study ${ }^{10}$. In our Monte Carlo simulation of regimen 1, we found that its hydroxychloroquine plasma concentrations with virological clearance achieved a \%PTA of only $23.5 \%$ and $46.1 \%$ at Days 5 and 10 , respectively. The poor performance of regimen 1 was consistent with the results of a separate clinical trial, which confirmed its lack of efficacy for adults ${ }^{34}$. By contrast, the results of the Monte Carlo simulation of the dosage regimen recommended by the Thai COVID-19 guidelines (regimen 4 in our study) were considerably better. That dosage is $10 \mathrm{mg} / \mathrm{kg} / \mathrm{dose}$ twice on Day 1 , followed by a $6.5 \mathrm{mg} / \mathrm{kg} /$ dose, twice daily, for the next 9 days. In our Monte Carlo simulation, this regimen demonstrated $>80 \%$ PTA after day 5 of treatment, and it achieved a plasma hydroxychloroquine level > than $0.83 \mathrm{mcg} / \mathrm{ml}$ for 10 days. These simulation findings are consistent with the good clinical outcomes that are found when the regimen is used in adults in Thailand. Unfortunately, regimen 4 exceeded the target peak hydroxychloroquine level that had been specified by our study $(2.6 \mathrm{mcg} / \mathrm{mL})$, with a \%PTA of $26.2 \%$ on day 10 of the treatment. Given that it took some time to achieve the therapeutic level and had a peak level that risked QT prolongation, regimen 4 might not be appropriate. However, the newest version of Thai COVID-19 guideline does not recommend using hydroxychloroquine for COVID-19 treatment. As to the recommended dosage regimen of the Pediatric Infectious Diseases Society ${ }^{16}$ (regimen 3:13 mg/kg/dose; then, 6.5 $\mathrm{mg} / \mathrm{kg} /$ dose at $6,24,48,72$, and 96 hours), it demonstrated a good safety profile (\%PTA of peak hydroxychloroquine level $<1 \%)$. However, it also had a low \%PTA $(<40 \%)$ to achieve the therapeutic plasma level (Ctrough $>0.83 \mathrm{mcg} /$ $\mathrm{mL}$ ) during the course of treatment. This represents a high risk of therapeutic failure. The results for the dosage regimen from the Gautret study ${ }^{3}$ (regimen 2) showed a \%PTA to achieve therapeutic target of $67.1 \%$ at day 6 of the treatment, and more than $80 \%$ at day 10 . These findings correspond with the PCR results (negative PCR $57.1 \%$ ) that the Gautret clinical trial found for adults after day 6 of being treated solely with hydroxychloroquine. They are also consistent with the very low probability of cardiovascular toxicity reported by Gautret and colleagues' adult clinical trial (\%PTA $<5 \%)$. Another study on the optimal hydroxychloroquine dosage regimen for pediatrics was undertaken by Maharaj et al. ${ }^{22}$. Their dosage (regimen 5 in our study) was derived from a physiologically based PK model that simulated dosages used in previous adult analyses. Their model showed that their dosage couldn't achieve the plasma concentration of hydroxychloroquine target from in vitro data on the effective concentration $\left(\mathrm{EC}_{50} ; 0.242 \mathrm{mcg} / \mathrm{mL}\right)$ value for treatment of COVID-19 infected Vero cell ${ }^{10}$. In our Monte Carlo simulation of regimen 5, the \%PTA of achieved therapeutic target was less than 50\% throughout the 10 days of treatment, but there was a high safety profile (\%PTA <1\%). Since the investigation by Maharaj and colleagues used simulation doses that were based on in vitro concentrations, the effects of their hydroxychloroquine dosage may not correlate with clinical outcomes.

The first limitation of our study was that our PK model relied upon data of healthy and malariainfected adult populations. This was due to the lack of pediatric studies. As the PK parameters that we chose were derived from a study cohort that was aged about 25 to 30 years and had standard body sizes, it is possible that any inaccuracies were reduced after allometric scaling. Moreover, differences in disease severity and age groups could affect some parts of our PK parameters, for example, GI absorption. Another point of concern was that hydroxychloroquine has a large volume of distribution, causing it to take a long time to reach a steady state. However, malaria and COVID-19 are acute diseases. Consequently, a non-steady state PK profile was deemed appropriate. Furthermore, our use of 0.75 as the allometric scaling exponent could introduce some errors ${ }^{18-20}$. On the other hand, a pediatric age (2-12 years) was used in our study; some PK properties (alpha 1-acid glycoprotein, renal function, and CYP enzyme activity) nearly reached adult values; and no significant genetic polymorphism has been reported. Therefore, this point might not be an issue ${ }^{35-38}$. The last limitation relates to the plasma hydroxychloroquine level that we used in this study. Gautret and colleagues ${ }^{3}$ did not report exactly when they collected the blood samples used to determine the average hydroxychloroquine plasma level needed for virological clearance in their clinical trial. We were therefore obliged to assume that they had collected plasma hydroxychloroquine immediately before administration of next doses, and that the plasma concentrations they reported were the trough levels. Another limitation of the present work was that it drew upon plasma concentration data from a clinical trial that had not only a small sample size, but also only adult patients.

One of the several strengths of this study is that the target trough level for hydroxychloroquine used in our simulation was obtained from a clinical trial. That trial measured the clinical outcomes, negative PCR on 
day 6 of treatment, and the hydroxychloroquine plasma concentration that correlated with the virological clearance of COVID-19. Another strength is that the dosage regimen which we have recommended would rapidly achieve a therapeutic level within 2 days of the commencement of treatment. This means that there will be a fast virological clearance. This will in turn contribute to a reduction in the transmission rates of COVID19 within hospitals as well as lessen the chance of its transmission to healthcare workers. Moreover, this is the first study to establish a single hydroxychloroquine dosing regimen that can be used for all pediatric ages between 2 and 12 years. This user-friendly regimen will therefore contribute to a reduction in medication errors. Finally, the low loading dose of the regimen means that a low peak hydroxychloroquine level is reached, thereby giving it a good safety profile.

\section{CONCLUSIONS}

The optimal dosage regimen of hydroxychloroquine is loading dosage of $6 \mathrm{mg} / \mathrm{kg} /$ dose every 6 hours for 2 days, followed by $3.25 \mathrm{mg} / \mathrm{kg} /$ dose every 8 hours on days 3-10. This regimen should rapidly achieve a therapeutic level, leading to early virological clearance and low cardiotoxicity in pediatric patients. However, clinical studies are required to confirm the efficacy and safety of this regimen.

\section{ACKNOWLEDGEMENT}

The authors thank Mr. David Park for English proofreading of the manuscript. This research did not receive any specific grant from funding agencies in the public, commercial, or not-for-profit sectors.

\section{Conflict of interest}

The authors declare that they have no conflict of interest.

\section{Funding}

None to declare.

\section{Ethics approval}

None to declare.

Article info:

Received June 29, 2021

Received in revised form September 24, 2021

Accepted October 29, 2021

\section{REFERENCES}

1. Dong Y, Mo X, Hu Y, Qi X, Jiang F, Jiang Z, et al. Epidemiology of COVID-19 Among Children in China. Pediatrics. 2020;145 (6):e20200702.

2. Qiu H, Wu J, Hong L, Luo Y, Song Q, Chen D. Clinical and epidemiological features of 36 children with coronavirus disease 2019 (COVID-19) in Zhejiang, China: an observational cohort study. Lancet Infect Dis. 2020;20(6):689-96.

3. Gautret P, Lagier JC, Parola P, Hoang VT, Meddeb L, Mailhe $\mathrm{M}$, et al. Hydroxychloroquine and azithromycin as a treatment of COVID-19: results of an open-label non-randomized clinical trial. Int J Antimicrob Agents. 2020;56(1):105949.

4. Chen Z, Hu J, Zhang Z, Jiang S, Han S, Yan D, et al. Efficacy of hydroxychloroquine in patients with COVID-19: results of a randomized clinical trial. medRxiv. 2020.

5. Arshad S, Kilgore P, Chaudhry ZS, Jacobsen G, Wang DD, Huitsing K, et al. Treatment with hydroxychloroquine, azithromycin, and combination in patients hospitalized with COVID-19. Int J Infect Dis. 2020;97:396-403.

6. Mikami T, Miyashita H, Yamada T, Harrington M, Steinberg D, Dunn A, et al. Risk Factors for Mortality in Patients with COVID-19 in New York City. J Gen Intern Med. 2021;36(1):17-26.

7. Tang W, Cao Z, Han M, Wang Z, Chen J, Sun W, et al. Hydroxychloroquine in patients with mainly mild to moderate coronavirus disease 2019: Open label, randomised controlled trial. BMJ. 2020;369:m1849.

8. Geleris J, Sun Y, Platt J, Zucker J, Baldwin M, Hripcsak G, et al. Observational Study of Hydroxychloroquine in Hospitalized Patients with Covid-19. N Engl J Med. 2020;382(25):2411-8.

9. Molina JM, Delaugerre C, Le Goff J, Mela-Lima B, Ponscarme D, Goldwirt L, et al. No evidence of rapid antiviral clearance or clinical benefit with the combination of hydroxychloroquine and azithromycin in patients with severe COVID-19 infection. Med Mal Infect. 2020;50(4):384.

10. Yao X, Ye F, Zhang M, Cui C, Huang B, Niu P, et al. In Vitro Antiviral Activity and Projection of Optimized Dosing Design of Hydroxychloroquine for the Treatment of Severe Acute Respiratory Syndrome Coronavirus 2 (SARS-CoV-2). Clin Infect Dis. 2020;71(15):732-9.

11. Liu J, Cao R, Xu M, Wang X, Zhang H, Hu H, et al. Hydroxychloroquine, a less toxic derivative of chloroquine, is effective in inhibiting SARS-CoV-2 infection in vitro. Cell Discov. 2020; 6(1):16.

12. Touret F, Gilles M, Barral K, Nougairède A, van Helden J, Decroly E, et al. In vitro screening of a FDA approved chemical library reveals potential inhibitors of SARS-CoV-2 replication. Sci Rep. 2020;10(1):13093.

13. Sanders JM, Monogue ML, Jodlowski TZ, Cutrell JB. Pharmacologic Treatments for Coronavirus Disease 2019 (COVID-19): A Review. JAMA. 2020;323(18):1824-36.

14. Ben-Zvi I, Kivity S, Langevitz P, Shoenfeld Y. Hydroxychloroquine: From Malaria to Autoimmunity. Clinic Rev Allerg Immunol. 2012;42(2):145-53.

15. Schrezenmeier E, Dörner T. Mechanisms of action of hydroxychloroquine and chloroquine: implications for rheumatology. Nat Rev Rheumatol. 2020;16(3):155-66.

16. Chiotos K, Hayes M, Kimberlin DW, Jones SB, James SH, Pinninti SG, et al. Multicenter Initial Guidance on Use of Antivirals for Children With Coronavirus Disease 2019/Severe Acute Respiratory Syndrome Coronavirus 2. J Pediatric Infect Dis Soc. 2020;9(6):701-15

17. Lim HS, Im JS, Cho JY, Bae KS, Klein TA, Yeom JS, et al. Pharmacokinetics of hydroxychloroquine and its clinical implications in chemoprophylaxis against malaria caused by Plasmodium vivax. Antimicrob Agents Chemother. 2009;53(4):1468-75.

18. Calvier EA, Krekels EH, Välitalo PA, Rostami-Hodjegan A, Tibboel D, Danhof M, et al. Allometric Scaling of Clearance in Paediatric Patients: When Does the Magic of 0.75 Fade?. Clin Pharmacokinet. 2017;56(3):273-85.

19. Mahmood I. Dosing in children: a critical review of the pharmacokinetic allometric scaling and modelling approaches in paediatric drug development and clinical settings. Clin Pharmacokinet. 2014;53(4):327-46.

20. Mahmood I, Staschen C-M, Goteti K. Prediction of drug clearance in children: an evaluation of the predictive performance of several models. AAPS J. 2014;16(6):1334-43. 
21. Holford N, Heo YA, Anderson B. A Pharmacokinetic Standard for Babies and Adults. J Pharm Sci. 2013;102(9):2941-52.

22. Maharaj AR, Wu H, Hornik CP, et al. Simulated Assessment of Pharmacokinetically Guided Dosing for Investigational Treatments of Pediatric Patients with Coronavirus Disease 2019. JAMA Pediatr. 2020;174(10):e202422.

23. Fernandez E, Perez R, Hernandez A, Tejada P, Arteta M, Ramos JT. Factors and Mechanisms for Pharmacokinetic Differences between Pediatric Population and Adults. Pharmaceutics. 2011; 3(1):53-72.

24. McChesney EW. Animal toxicity and pharmacokinetics of hydroxychloroquine sulfate. Am J Med. 1983;75(1):11-8.

25. Jordan P, Brookes JG, Nikolic G, Le Couteur DG. Hydroxychloroquine overdose: toxicokinetics and management. J Toxicol Clin Toxicol. 1999;37(7):861-4.

26. Garcia-Cremades M, Solans BP, Hughes E, Ernest JP, Wallender E, Aweeka F, et al. Optimizing Hydroxychloroquine Dosing for Patients With COVID-19: An Integrative Modeling Approach for Effective Drug Repurposing. Clin Pharmacol Ther. 2020;108(2): 253-63.

27. Ursing J, Rombo L, Eksborg S, Larson L, Bruvoll A, Tarning J, et al. High-Dose Chloroquine for Uncomplicated Plasmodium falciparum Malaria Is Well Tolerated and Causes Similar QT Interval Prolongation as Standard-Dose Chloroquine in Children. Antimicrob Agents Chemother. 2020;64(3):e01846-19.

28. The United States Food and Drug Administration. A summary of the FDA review of safety issues with the use of hydroxychloroquine and chloroquine to treat hospitalized patients with COVID-19 s [document on the internet]. 2020 [cited 2020 July 12]. Available from: https://www.fda.gov/drugs/drug-safety-andavailability/fda-cautions-against-use-hydroxychloroquine-or-chloroquine-covid-19-outside-hospital-setting-or.
29. Sridhar AR, Chatterjee NA, Saour B, Nguyen D, Starnes EA, Johnston C, et al. QT interval and arrhythmic safety of hydroxychloroquine monotherapy in coronavirus disease 2019. Heart Rhythm O2. 2020;1(3):167-72.

30. Marquardt K, Albertson TE. Treatment of hydroxychloroquine overdose. Am J Emerg Med. 2001;19(5):420-4.

31. Kemmenoe AV. An infant fatality due to hydroxychloroquine poisoning. J Anal Toxicol. 1990;14(3):186-8.

32. Olano DJ, Howland MA, Su MK, Hoffman RS, Biary R. Toxicokinetics of hydroxychloroquine following a massive overdose. Am J Emerg Med. 2019;37(12):2264.e5-8.

33. Mercuro NJ, Yen CF, Shim DJ, Maher TR, McCoy CM, Zimetbaum PJ, et al. Risk of QT Interval Prolongation Associated With Use of Hydroxychloroquine With or Without Concomitant Azithromycin Among Hospitalized Patients Testing Positive for Coronavirus Disease 2019 (COVID-19). JAMA Cardiol. 2020;5(9): 1036-41.

34. Self WH, Semler MW, Leither LM, Casey JD, Angus DC, Brower RG, et al. Effect of Hydroxychloroquine on Clinical Status at 14 Days in Hospitalized Patients With COVID-19: A Randomized Clinical Trial. JAMA. 2020;324(21):2165-76.

35. Tett S, Cutler D, Day R, Brown K. Bioavailability of hydroxychloroquine tablets in healthy volunteers. Br J Clin Pharmacol. 1989;27(6):771-9.

36. Kodidela S, Kumar S, Uppugunduri C. Developmental pattern of hepatic drug-metabolizing enzymes in pediatric population and its role in optimal drug treatment. Arch Med Health Sci. 2017;5(1):115-22.

37. Gil J, Berglund EG. CYP2C8 and antimalaria drug efficacy. Pharmacogenomics. 2007;8(2):187-98.

38. Smith SA, Waters NJ. Pharmacokinetic and Pharmacodynamic Considerations for Drugs Binding to Alpha-1-Acid Glycoprotein. Pharm Res. 2018;36(2):30. 